\title{
Integral Baire-1 Stieltjes, Henstock-Stieltjes dan Riemann-Stieltjes
}

\author{
Kalfin D. Muchtar ${ }^{1}$, Jullia Titaley ${ }^{2}$, Mans L. Mananohas ${ }^{3}$ \\ ${ }^{1}$ Program Studi Matematika, FMIPA, UNSRAT Manado, kalfin_muchtar@yahoo.com \\ ${ }^{2}$ Program Studi Matematika, FMIPA, UNSRAT Manado, july titaley@yahoo.com \\ ${ }^{3}$ Program Studi Matematika, FMIPA, UNSRAT Manado, mansmananohas@ yahoo.com
}

\begin{abstract}
Abstrak
Beberapa sifat dasar termasuk Kriteria Cauchy dan Teorema Aditif dapat diberlakukan pada konsep integral Baire-1 Stieltjes. Misalkan $f$ dan $g$ merupakan fungsi-fungsi bernilai real yang didefinisikan pada $[a, b] \subset \mathbb{R}$. Jika $f$ terintegral Baire-1 Stieltjes terhadap $g$ pada $[a, b]$, maka $f$ terintegral Henstock-Stieltjes terhadap $g$ pada $[a, b]$ dengan nilai integralnya sama. Syarat cukup agar fungsi $f$ yang terintegral Henstock-Stieltjes terhadap $g$ pada $[a, b]$ terintegral Baire-1 Stieltjes terhadap $g$ pada $[a, b]$ yaitu $f$ fungsi kelas Baire-1 dan $g$ fungsi bervariasi terbatas pada $[a, b]$. Jika $f$ terintegral Riemann-Stieltjes terhadap fungsi $g$ pada $[a, b]$, maka $f$ terintegral Baire-1 Stieltjes terhadap $g$ pada $[a, b]$ dengan nilai integralnya sama.
\end{abstract}

Kata Kunci: Integral Baire-1 Stieltjes, Integral Henstock-Stieltjes, Integral Riemann-Stieltjes

\section{The Stieltjes Integrals of Baire-1, Henstock and Riemann}

\begin{abstract}
The Baire-1 Stieltjes integral possess the elementary integral properties including Cauchy Criterion and Additivity Theorem. Let $f$ and $g$ are real valued functions defined on $[a, b] \subset \mathbb{R}$. If $f$ is Baire-1 Stieltjes integrable with respect to $g$ on $[a, b]$, then $f$ is Henstock-Stieltjes integrable with respect to $g$ on $[a, b]$, and the values of the integrals are the same. We obtain that the integrand which is Baire-1 function and the integrator which is of bounded variation is a sufficient condition for Henstock-Stieltjes integrable function to be Baire-1 Stieltjes integrable. If $f$ is Riemann-Stieltjes integrable with respect to $g$ on $[a, b]$, then $f$ is Baire-1 Stieltjes integrable with respect to $g$ on $[a, b]$, and the values of the integrals are the same.
\end{abstract}

Keywords: Baire-1 Stieltjes Integral, Henstock-Stieltjes Integral, Riemann-Stieltjes Integral

\section{Pendahuluan}

Integral tipe Stieltjes merupakan pengembangan integral biasa dalam artian panjang $v-u$ dari subinterval $[u, v]$ yang digunakan pada definisi integral biasa diganti dengan $g(v)-g(u)$ untuk suatu fungsi $g$ bernilai real. Integral Riemann-Stieltjes merupakan integral tipe Stieltjes yang pertama kali diperkenalkan oleh Thomas Joannes Stieltjes pada tahun 1894. Kemudian pada tahun 1914, Frigyes Riesz menggunakan teori integral Riemann-Stieltjes untuk menunjukkan bahwa setiap fungsional linier pada ruang fungsi kontinu dapat diekspresikan menggunakan integral tersebut. Teorema ini kemudian dikenal sebagai Teorema Representasi Riesz [1] .

Pada tahun 1998 Jong Sul Lim, Ju Han Yoon dan Gwang Sik Eun [2] mendefinisikan integral Henstock-Stieltjes fungsi bernilai real dan menyelidiki beberapa sifat dasar dari integral tipe Stieltjes ini. Pada tahun 2001 Lee dan Caroline Su Yin [3] mendefinisikan suatu jenis integral tipe Riemann yang belakangan dikenal sebagai integral Baire-1dan menyelidiki sifat-sifat dasarnya serta mengkaji hubungan antara integral Baire-1 dengan integral Riemann dan integral HenstockKurzweil.

Pada tahun 2014, Karlo S. Orge dan Julius V. Benitez [1] mengembangkan definisi integral Baire-1 tipe Stieltjes. Dalam makalah ini, dibuktikan beberapa sifat dasar integral Baire-1 Stieltjes, di antaranya Kriteria Cauchy dan Teorema Aditif. Selain itu telah dikaji hubungan integral Henstock-Stieltjes dan integral Baire-1 Stieltjes, di antaranya dengan memberikan syarat cukup agar fungsi yang terintegral Henstock-Stieltjes terintegral Baire-1 Stieltjes, dan dikaji pula hubungan antara integral Riemann-Stieltjes dan integral Baire-1 Stieltjes. 


\section{Fungsi Baire-1}

Definisi 1 [4]

Diberikan fungsi $f: \mathbb{R} \rightarrow \mathbb{R} . f$ disebut fungsi Baire-1 jika ada barisan fungsi kontinu yang konvergen titik demi titik (pointwise convergence) ke $f$.

Definisi 2 [5]

Suatu fungsi $f: \mathbb{R} \rightarrow \mathbb{R}$ disebut fungsi Baire-1 jika $\forall \epsilon>0$ terdapat fungsi positif $\delta(\cdot)$ pada $\mathbb{R}$ sedemikian sehingga $\forall x, y \in$ dengan $|x-y|<\min \{\delta(x), \delta(y)\}$ berakibat $|f(x)-f(y)|<\epsilon$.

\section{Partisi Terlabel dan Gauge pada $[a, b]$}

Definisi 3 [6]

Diberikan suatu himpunan tertutup dan terbatas $I:=[a, b] \subset \mathbb{R}$.

a. Sebuah partisi (partition) dari interval $I$ didefinisikan sebagai koleksi $\mathcal{P}:=\left\{I_{i}\right\}_{i=1}^{n}$ sedemikian sehingga $\operatorname{int}\left(I_{i}\right) \cap \operatorname{int}\left(I_{j}\right)=\emptyset$ dan $\bigcup_{i=1}^{n} I_{i}=I$ diman $I_{i}:=\left[x_{i-1}, x_{i}\right]$ dan $a=x_{0}<\cdots<x_{i-1}<x_{i}<\cdots x_{n}=b$.

b. Didefinisikan norm pada $\mathcal{P}$ yaitu $\|\mathcal{P}\|:=\sup _{1 \leq i \leq n}\left(x_{i}-x_{i-1}\right)$.

c. Titik $x_{i} \forall i \in\{1, \ldots, n\}$ disebut sebagai titik partisi (partition point) dari $\mathcal{P}$.

d. Sebuah titik $\xi_{i}$ yang dipilih dari interval $I_{i} \forall i \in\{1, \ldots, n\}$ disebut sebagai label (tag), dan koleksi $\dot{\mathcal{P}}:=\left\{\left(\left[x_{i-1}, x_{i}\right], \xi_{i}\right)\right\}_{i=1}^{n}$ disebut sebagai partisi terlabel (tagged partition) dari $I$.

\section{Fungsi Bervariasi Terbatas}

\section{Definisi 4 [7]}

Diberikan fungsi $f:[a, b] \rightarrow \mathbb{R}$. Variasi dari $f$ pada $[a, b]$ didefinisikan sebagai $\operatorname{Var}(f,[a, b])=\sup \left\{\sum_{i=1}^{n}\left|f\left(x_{i}\right)-f\left(x_{i-1}\right)\right|: \mathcal{P}\right.$ partisi dari $\left.[a, b]\right\}$.

Jika ada bilangan real $M>0$ sedemikian sehingga $\operatorname{Var}(f,[a, b]) \leq M$, maka $f$ fungsi bervariasi terbatas pada $[a, b]$.

\section{Integral Riemann-Stieltjes}

\section{Definisi 5 [7]}

Diberikan dua fungsi $f, g:[a, b] \rightarrow \mathbb{R}$. Fungsi $f$ terintegral Riemann-Stieltjes terhadap $g$ pada $[a, b]$ jika ada bilangan real $L$ sedemikian sehingga $\forall \epsilon>0$ terdapat bilangan real $\delta>0$ sedemikian sehingga jika $\dot{\mathcal{P}}$ sebarang partisi terlabel dengan $\|\mathcal{P}\|<\delta$, maka

dimana $S(f, g, \dot{\mathcal{P}})=\sum_{i=1}^{n} f\left(\xi_{i}\right)\left(g\left(x_{i}\right)-g\left(x_{i-1}\right)\right)$.

$$
|S(f, g, \dot{\mathcal{P}})-L|<\epsilon
$$

Lebih lanjut, bilangan real $L$ disebut sebagai integral Riemann-Stieltjes $f$ terhadap $g$ pada $[a, b]$ dan ditulis

$$
L=(\mathcal{R S}) \int_{a}^{b} f d g .
$$

\section{Integral Henstock-Stieltjes}

\section{Definisi 6 [2]}

Misalkan $g$ fungsi monoton naik pada $[a, b]$. Fungsi $f:[a, b] \rightarrow \mathbb{R}$ terintegral Henstock-Stieltjes terhadap $g$ pada $[a, b]$ jika ada bilangan real $L$ sedemikian sehingga $\forall \epsilon>0$ terdapat gauge $\delta(\cdot)$ pada $[a, b]$ sedemikian sehingga jika $\dot{\mathcal{P}}$ sebarang partisi terlabel yang subordinat terhadap $\delta(\cdot)$, maka

$$
|S(f, g, \dot{\mathcal{P}})-L|<\epsilon
$$

dimana $S(f, g, \dot{\mathcal{P}})=\sum_{i=1}^{n} f\left(\xi_{i}\right)\left(g\left(x_{i}\right)-g\left(x_{i-1}\right)\right)$.

Lebih lanjut, bilangan real $L$ disebut sebagai integral Henstock-Stieltjes $f$ terhadap $g$ pada $[a, b]$ dan ditulis $L=(\mathcal{H} \mathcal{S}) \int_{a}^{b} f d g$. 


\section{Integral Baire-1 Stieltjes}

Definisi 7 [1]

Misalkan $\delta(\cdot)$ gauge pada $[a, b]$. Misalkan pula $\dot{\mathcal{P}}=\left\{\left(\left[x_{i-1}, x_{i}\right], \xi_{i}\right)\right\}_{i=1}^{n}$ dan $\dot{\mathcal{P}}^{*}=\left\{\left(\left[x_{i-1}, x_{i}\right], \eta_{i}\right)\right\}_{i=1}^{n}$ partisi terlabel dari $[a, b]$. Partisi terlabel $\dot{\mathcal{P}}^{*}$ dikatakan sebagai $\dot{\mathcal{P}}$-compatible dan ditulis $\dot{\mathcal{P}}^{*} \sim \dot{\mathcal{P}}$ jika $\left|\xi_{i}-\eta_{i}\right|<\delta\left(\eta_{i}\right) \forall i \in\{1, \ldots, n\}$.

\section{Definisi 8 [1]}

Diberikan dua fungsi $f, g:[a, b] \rightarrow \mathbb{R}$. Fungsi $f$ terintegral Baire-1 Stieltjes terhadap $g$ pada $[a, b]$ jika ada bilangan real $L$ sedemikian sehingga $\forall \epsilon>0$ terdapat gauge $\delta(\cdot)$ pada $[a, b]$ sedemikian sehingga jika $\dot{\mathcal{P}}$ sebarang partisi terlabel yang subordinat terhadap $\delta(\cdot)$ dan $\dot{\mathcal{P}}^{*}$ sebarang partisi terlabel dengan $\dot{\mathcal{P}}^{*} \sim \dot{\mathcal{P}}$, maka

dimana

$$
\left|S\left(f, g, \dot{\mathcal{P}}^{*}\right)-L\right|<\epsilon
$$

$$
S\left(f, g, \dot{\mathcal{P}}^{*}\right)=\sum_{i=1}^{n} f\left(\eta_{i}\right)\left(g\left(x_{i}\right)-g\left(x_{i-1}\right)\right) .
$$

Lebih lanjut, bilangan real $L$ disebut sebagai integral Baire-1 Stieltjes $f$ terhadap $g$ pada $[a, b]$ dan ditulis

$$
L=(\mathcal{B S}) \int_{a}^{b} f d g .
$$

\section{Metodologi Penelitian}

Metode yang digunakan dalam penelitian ini adalah dengan melakukan studi literatur dengan tahapan sebagai berikut :

a. Mempelajari konsep integral Baire-1 Stieltjes, integral Henstock-Stieltjes dan integral Riemann-Stieltjes.

b. Mempelajari konsep fungsi Baire-1.

c. Membuktikan beberapa sifat dasar integral Baire-1 Stieltjes.

d. Membuktikan bahwa setiap fungsi terintegral Baire-1 Stieltjes terintegral Henstock-Stieltjes.

e. Membangun syarat cukup agar fungsi yang terintegral Henstock-Stieltjes terintegral Baire-1 Stieltjes.

f. Membuktikan bahwa setiap fungsi yang terintegral Riemann-Stieltjes terintegral Baire-1 Stieltjes.

\section{Hasil dan Pembahasan}

\subsection{Beberapa Sifat Dasar Integral Baire-1 Stieltjes}

\section{Teorema 1 [1]}

Misalkan $f, g:[a, b] \rightarrow \mathbb{R}$. Jika $f$ terintegral Baire-1 Stieltjes terhadap fungsi $g$ pada $[a, b]$, maka integralnya tunggal.

\section{Teorema 2 [1]}

Misalkan $f$ dan $f^{*}$ terintegral Baire-1 Stieltjes terhadap $g$ pada $[a, b]$ dan $f$ terintegral Baire-1 Stieltjes terhadap $g^{*}$ pada $[a, b]$. Misalkan $\alpha \in \mathbb{R}$. Maka

a. $f+f^{*}$ terintegral Baire-1 Stieltjes terhadap $g$ pada $[a, b]$, dan

$$
(\mathcal{B S}) \int_{a}^{b}\left(f+f^{*}\right) d g=(\mathcal{B} \mathcal{S}) \int_{a}^{b} f d g+(\mathcal{B S}) \int_{a}^{b} f^{*} d g .
$$

b. $\quad \alpha f$ terintegral Baire-1 Stieltjes terhadap $g$ pada $[a, b]$, dan

$$
(\mathcal{B S}) \int_{a}^{b} \alpha f d g=\alpha \cdot(\mathcal{B S}) \int_{a}^{b} f d g .
$$

c. $f$ terintegral Baire-1 Stieltjes terhadap $g+g^{*}$ pada $[a, b]$, dan

$$
(\mathcal{B S}) \int_{a}^{b} f d\left(g+g^{*}\right)=(\mathcal{B S}) \int_{a}^{b} f d g+(\mathcal{B S}) \int_{a}^{b} f d g^{*}
$$

d. $f$ terintegral Baire-1 Stieltjes terhadap $\alpha g$ pada $[a, b]$, dan

$$
(\mathcal{B S}) \int_{a}^{b} f d \alpha g=\alpha \cdot(\mathcal{B S}) \int_{a}^{b} f d g .
$$




\section{Teorema 3 [1]}

Misalkan $f, g:[a, b] \rightarrow \mathbb{R}$. Maka $f$ terintegral Baire-1 Stieltjes terhadap $g$ pada $[a, b]$ jika dan hanya jika $\forall \epsilon>0$, terdapat suatu gauge $\delta(\cdot)$ pada $[a, b]$ sedemikian sehingga untuk setiap dua partisi terlabel $\dot{\mathcal{P}}$ dan $\dot{\mathcal{Q}}$ yang subordinat terhadap $\delta(\cdot)$ dan untuk semua $\dot{\mathcal{P}}^{*} \sim \dot{\mathcal{P}}$ dan $\dot{\mathcal{Q}}^{*} \sim \dot{\mathcal{Q}}$, maka

$$
\left|S\left(f, g, \dot{\mathcal{P}}^{*}\right)-S\left(f, g, \dot{\mathcal{Q}}^{*}\right)\right|<\epsilon .
$$

\section{Teorema 4 [1]}

Misalkan $f, g:[a, b] \rightarrow \mathbb{R}$ dan $c \in(a, b)$. Jika $f$ terintegral Baire-1 Stieltjes terhadap $g$ pada $[a, c]$ dan $[a, c]$, maka $f$ terintegral Baire-1 Stieltjes terhadap $g$ pada $[a, b]$, dan

$$
(\mathcal{B S}) \int_{a}^{b} f d g=(\mathcal{B S}) \int_{a}^{c} f d g+(\mathcal{B S}) \int_{c}^{b} f d g .
$$

\section{Teorema 5}

Diberikan fungsi-fungsi $f, g, h:[a, b] \rightarrow \mathbb{R}$. Misalkan $g$ monoton naik pada $[a, b]$ dan $f(x) \leq$ $h(x) \forall x \in[a, b]$. Jika $f$ dan $h$ masing-masing terintegral Baire-1 Stieltjes terhadap $g$ pada $[a, b]$, maka

$$
(\mathcal{B S}) \int_{a}^{b} f d g \leq(\mathcal{B S}) \int_{a}^{b} h d g .
$$

\subsection{Hubungan Integral Henstock-Stieltjes dan Integral Baire-1 Stieltjes}

\section{Teorema 6}

Jika $f$ terintegral Baire-1 Stieltjes terhadap $g$ pada $[a, b]$, maka $f$ terintegral Henstock-Stieltjes terhadap $g$ pada $[a, b]$ dan

$$
(\mathcal{B S}) \int_{a}^{b} f d g=(\mathcal{H} \mathcal{S}) \int_{a}^{b} f d g
$$

\section{Teorema 7}

Misalkan $f$ terintegral Henstock-Stieltjes terhadap $g$ pada $[a, b]$. Jika $f$ fungsi Baire-1 dan $g$ bervariasi terbatas pada $[a, b]$, maka $f$ terintegral Baire-1 Stieltjes terhadap $g$ pada $[a, b]$.

Contoh :

Diberikan dua fungsi $f, g:[1,2] \rightarrow \mathbb{R}$ yang masing-masing didefinisikan sebagai

$$
f(x):=\left\{\begin{array}{ll}
1, & x \in\left\{\frac{3}{2}, \frac{7}{4}\right\} \\
0 & , x \in[1,2] \backslash\left\{\frac{3}{2}, \frac{7}{4}\right\}
\end{array} \text { dan } g(x):=x^{2} \forall x \in[1,2] .\right.
$$

Buktikan bahwa $(\mathcal{B S}) \int_{1}^{2} f d g=0$.

Solusi :

Diberikan sebarang $\epsilon>0$. Pertama akan ditunjukkan bahwa $g$ fungsi bervariasi terbatas pada $[1,2]$. Karena $g \in C^{1}[1,2]$ monoton naik, maka $g$ dan $g^{\prime}$ kontinu pada [1,2]. Akibatnya $g^{\prime}$ terbatas pada [1,2], yakni terdapat bilangan real $M>0$ sedemikian sehingga $\left|g^{\prime}(x)\right| \leq M \forall x \in[1,2]$. Akibatnya $g$ bervariasi terbatas pada $[1,2]$ dengan

$$
\begin{aligned}
\operatorname{Var}(g,[a, b]) & =\sup \left\{\sum_{\mathrm{i}=1}^{\mathrm{n}}\left|g\left(x_{i}\right)-g\left(x_{i-1}\right)\right|: \dot{\mathcal{P}} \text { partisi dari }[1,2] .\right\} \\
& =g(b)-g(a)=b^{2}-a^{2} .
\end{aligned}
$$

Selanjutnya akan ditunjukkan bahwa $f$ fungsi Baire-1. Misalkan $A:=\left\{\frac{3}{2}, \frac{7}{4}\right\}$. Karena $f$ kontinu di setiap $\xi \in[1,2] \backslash\left\{\frac{3}{2}, \frac{7}{4}\right\}$, maka terdapat bilangan real $\delta_{\xi}>0$ sedemikian sehingga $\forall x \in[1,2]$ dengan $|x-\xi|<\delta_{\xi}$, maka $|f(x)-f(\xi)|<\frac{\epsilon}{2\left(b^{2}-a^{2}\right)}$. Sekarang definisikan fungsi positif $\delta(\cdot)$ pada $[1,2]$ dengan rumus

$$
\delta(x):= \begin{cases}\frac{1}{8}, & x \in\left\{\frac{3}{2}, \frac{7}{4}\right\} \\ \delta_{x}, & x \in[1,2] \backslash\left\{\frac{3}{2}, \frac{7}{4}\right\}\end{cases}
$$

Misalkan $|x-y|<\min \{\delta(x), \delta(y)\}$. Maka hanya ada tiga kemungkinan.

Pertama, $x \in[1,2] \backslash\left\{\frac{3}{2}, \frac{7}{4}\right\}$ dan $y \in\left\{\frac{3}{2}, \frac{7}{4}\right\}$. Maka $|x-y|<\min \left\{\delta_{x}, \frac{1}{8}\right\}<\delta_{x}$.

Oleh karena itu $|f(x)-f(y)|<\frac{\epsilon}{2\left(b^{2}-a^{2}\right)}$. Kedua, $x \in\left\{\frac{3}{2}, \frac{7}{4}\right\}$ dan $y \in[1,2] \backslash\left\{\frac{3}{2}, \frac{7}{4}\right\}$. 
Maka $|x-y|<\min \left\{\frac{1}{8}, \delta_{y}\right\}<\delta_{y}$. Oleh karena itu $|f(x)-f(y)|<\frac{\epsilon}{2\left(b^{2}-a^{2}\right)}$.

Ketiga, $x, y \in[1,2] \backslash\left\{\frac{3}{2}, \frac{7}{4}\right\}$. Maka $|x-y|<\min \left\{\delta_{x}, \delta_{y}\right\}<\delta_{x}$.

Oleh karena itu $|f(x)-f(y)|<\frac{\epsilon}{2\left(b^{2}-a^{2}\right)}$. Berdasarkan ketiga kemungkinan tersebut, maka dapat disimpulkan bahwa $f$ fungsi kelas Baire-1. Selanjutnya, definisikan gauge $\rho(\cdot)$ pada $[1,2]$ dengan rumus

$$
\rho(x):=\left\{\begin{aligned}
\frac{\epsilon}{16 M}, & x \in\left\{\frac{3}{2}, \frac{7}{4}\right\} \\
1, & x \in[1,2] \backslash\left\{\frac{3}{2}, \frac{7}{4}\right\}
\end{aligned}\right.
$$

Misalkan $\dot{\mathcal{P}}:=\left\{\left(\left[x_{i-1}, x_{i}\right], \xi_{i}\right)\right\}_{i=1}^{n}$ sebarang partisi terlabel yang subordinat terhadap $\rho(\cdot)$. Karena $g \in C^{1}[1,2]$, maka $\forall i \in\{1, \ldots, n\} \quad \exists c_{i} \in\left(x_{i-1}, x_{i}\right)$ sedemikian sehingga $g\left(x_{i}\right)-g\left(x_{i-1}\right)=g^{\prime}\left(c_{i}\right)\left(x_{i}-x_{i-1}\right)$.

Misalkan $\sigma:=\left\{i: \xi_{i} \in\left\{\frac{3}{2}, \frac{7}{4}\right\}\right\}$. Maka

$$
\begin{aligned}
|S(f, g, \dot{\mathcal{P}})|=\left|\sum_{i \in \sigma} f\left(\xi_{i}\right)\left(g\left(x_{i}\right)-g\left(x_{i-1}\right)\right)\right| & =\left|\sum_{i \in \sigma} g^{\prime}\left(c_{i}\right)\left(x_{i}-x_{i-1}\right)\right| \\
& \leq M \cdot\left|\sum_{i \in \sigma}\left(x_{i}-x_{i-1}\right)\right| \\
& <M \cdot 4 \cdot 2 \rho\left(\frac{3}{2}\right)=\frac{\epsilon}{2} .
\end{aligned}
$$

Sekarang definisikan sebuah fungsi positif $\gamma(\cdot):=\min \{\rho(\cdot), \delta(\cdot)\}$ pada $[1,2]$. Jelas bahwa $\gamma(\cdot)$ merupakan gauge pada [1,2]. Misalkan $\dot{\mathcal{Q}}:=\left\{\left(\left[y_{i-1}, y_{i}\right], \eta_{i}\right)\right\}_{i=1}^{n}$ sebarang partisi yang subordinat terhadap $\gamma(\cdot)$. Maka $\dot{\mathcal{Q}}$ juga subordinat terhadap $\rho(\cdot)$. Jika $\dot{\mathcal{Q}}^{*}:=\left\{\left(\left[y_{i-1}, y_{i}\right], \zeta_{i}\right)\right\}_{i=1}^{n}$ sebarang partisi terlabel dari $[1,2]$ dengan $\dot{Q}^{*} \sim \dot{\mathcal{Q}}$, maka

$$
\begin{aligned}
\left|S\left(f, g, \dot{Q}^{*}\right)\right| & =\left|S\left(f, g, \dot{Q}^{*}\right)-S(f, g, \dot{\mathcal{Q}})+S(f, g, \dot{\mathcal{Q}})\right| \\
& \leq\left|S\left(f, g, \dot{Q}^{*}\right)-S(f, g, \dot{\mathcal{Q}})\right|+|S(f, g, \dot{\mathcal{Q}})| \\
& =\left|\sum_{i=1}^{n}\left(f\left(\zeta_{i}\right)-f\left(\eta_{i}\right)\right)\left(g\left(y_{i}\right)-g\left(y_{i-1}\right)\right)\right|+|S(f, g, \dot{\mathcal{Q}})| \\
& <\frac{\epsilon}{2\left(b^{2}-a^{2}\right)} \sum_{i=1}^{n}\left(g\left(y_{i}\right)-g\left(y_{i-1}\right)\right)+\frac{\epsilon}{2} \\
& <\frac{\epsilon}{2}+\frac{\epsilon}{2}=\epsilon .
\end{aligned}
$$

Karena $\epsilon>0$ sebarang, maka dapat disimpulkan bahwa $f$ terintegral Baire-1 Stieltjes terhadap $g$ pada $[1,2]$ dengan $(\mathcal{B S}) \int_{1}^{2} f d g=0$.

\subsection{Hubungan Integral Riemann-Stieltjes dan Integral Baire-1 Stieltjes}

\section{Teorema 8}

Jika $f$ terintegral Riemann-Stieltjes terhadap $g$ pada $[a, b]$, maka $f$ terintegral Baire-1 Stieltjes terhadap $g$ pada $[a, b]$ dan

$$
(\mathcal{R S}) \int_{a}^{b} f d g=(\mathcal{B S}) \int_{a}^{b} f d g .
$$

\section{Lemma 1}

Misalkan $f:[a, b] \rightarrow \mathbb{R}$ terbatas dan $g:[a, b] \rightarrow \mathbb{R}$ monoton naik. Maka $f$ terintegral RiemannStieltjes terhadap fungsi $g$ pada $[a, b]$ jika dan hanya jika $\forall \epsilon>0$ ada partisi $\mathcal{P}$ dari $[a, b]$ sedemikian sehingga

$$
\mathcal{U}(f, g, \mathcal{P})-\mathcal{L}(f, g, \mathcal{P})<\epsilon .
$$

\section{Teorema 9}

Diberikan fungsi-fungsi $f, g:[a, b] \rightarrow \mathbb{R}$. Misalkan $f$ terbatas dan $g \in C^{1}[a, b]$ monoton naik pada $[a, b]$. Jika $f$ kontinu hampir di mana-mana pada $[a, b]$, maka $f$ terintegral Baire-1 Stieltjes terhadap $g$ pada $[a, b]$.

\section{Teorema 10}

Diberikan fungsi-fungsi $f, g:[a, b] \rightarrow \mathbb{R}$. Jika $f$ kontinu pada $[a, b]$ dan $g$ bervariasi terbatas pada $[a, b]$, maka $f$ terintegral Baire-1 Stieltjes terhadap $g$ pada $[a, b]$. 


\section{Kesimpulan}

Berdasarkan hasil dan pembahasan dapat ditarik kesimpulan :

a. Beberapa sifat dasar integral dapat diberlakukan pada konsep integral Baire-1 Stieltjes.

b. Jika $f$ sebarang fungsi yang terintegral Baire-1 Stieltjes terhadap suatu fungsi $g$ pada $[a, b]$, maka $f$ terintegral Henstock-Stieltjes terhadap $g$ pada $[a, b]$ dengan nilai integralnya sama. Lebih lanjut, jika $f$ terintegral Henstock-Stieltjes terhadap $g$ pada $[a, b], f$ fungsi kelas Baire-1 dan $g$ fungsi bervariasi terbatas pada $[a, b]$, maka $f$ terintegral Baire-1 Stieltjes terhadap $g$ pada $[a, b]$ dengan nilai integralnya sama.

c. Jika $f$ sebarang fungsi yang terintegral Riemann-Stieltjes terhadap suatu fungsi $g$ pada $[a, b]$, maka $f$ terintegral Baire-1 Stieltjes terhadap $g$ pada $[a, b]$ dengan nilai integralnya sama.

\section{Daftar Pustaka}

[1] Ogre, K. S., and J. V Benitez. 2014. Baire One-Stieltjes Integration., Int. Journal of Math. Analysis. 8(30) : 1465-1474.

[2] Lim, J. S., J.H Yoon., and G.S Eun.1998. On Henstock Stieltjes Integral. Kangweon-Kyungki Math. Jour. 6(1) : 87-96.

[3] Lee., C. S. Y. 2001. On Baire-1 Functions. Academic Excercise. National Institute of Education, Nanyang Technlogical University, Singapore. https://repository.nie.edu.sg/bitstream/10497/2383/3/LeeCarolineSuYin.html [23 Februari 2016].

[4] Fung, S. 2013. Functions of Baire Class One. Notes. Department of Mathematics, University of California, San Diego.

[5] Fenecios, J.P., and E. A Cabral. 2013. On Some Properties of Baire-1 Functions., Int. Journal of Math. Analysis.7(8): 393-402.

[6] Bartle, R.G., and D. R. Sherbert. 2011. Introduction to Real Analysis. $4^{\text {th }}$ Edition. John Wiley and Sons, New York.

[7] Protter, M. H. 1998. Basic Elements of Real Analysis. Springer. New York.

[8] Ghorpade, S. R., and B.V. Limaye. 2006. A Course in Calculus and Real Analysis. Springer, New York.

[9] Gunawan, H. 2009. Pengantar Analisis Real. FMIPA ITB, Bandung.

[10] Munkres, J. R. 2000. Topology. $2^{\text {nd }}$ Edition. Prentice Hall, Upper Saddle River. 